Article

\title{
Analysis of Subharmonic Oscillation and Slope Compensation for a Differential Boost Inverter
}

\author{
Abdelali El Aroudi ${ }^{1, *}{ }^{(i)}$, Mohamed Al-Numay ${ }^{2}$ (i), Reham Haroun ${ }^{1}$ (i) and Meng Huang ${ }^{3}$ \\ 1 Department of Electronics, Electrical Engineering and Automatic Control, Universitat Rovira i Virgili, \\ 43002 Tarragona, Spain; reham.haroun@urv.cat \\ 2 Electrical Engineering Department, King Saud University, 11451 Riyadh, Saudi Arabia; alnumay@ksu.edu.sa \\ 3 School of Electrical Engineering, Wuhan University, Wuhan 430072, China; meng.huang@whu.edu.cn \\ * Correspondence: abdelali.elaroudi@urv.cat; Tel.: +34-977-558-522
}

Received: 10 July 2020; Accepted: 11 August 2020; Published: 13 August 2020

\begin{abstract}
This paper focuses on the steady-behavior of a differential boost inverter used for generating a sinewave AC voltage in rural areas. The analysis of its dynamics will be performed using an accurate approach based on discrete time models and Floquet theory and adopting a quasi-static approximation. In particular, the undesired subharmonic oscillation exhibited by the inverter will be analyzed and its boundary in the parameter space will be predicted and delimited. Combining analytical expressions and computational procedures to determine the quasi-static duty cycle, subharmonic oscillation is accurately predicted. It is found that subharmonic oscillation takes place at critical values of the sinewave voltage reference cycle, which can cause distortion to the input current and degrade the harmonic content of the output voltage. The results provide useful information for the design of the boost inverter to avoid distortion caused by subharmonic oscillation. Namely, the minimum value of the compensation slope and the maximum proportional gain of the AC output voltage controller guaranteeing a pure sinewave voltage and clean inductor current during the entire AC cycle will be determined. Numerical simulations performed on the switched model implemented using PSIM(C) software confirm the theoretical predictions.
\end{abstract}

Keywords: differential boost inverter; current mode control; nonlinear behavior; subharmonic oscillation; slope compensation

\section{Introduction}

DC-AC inverters find widespread usage in many residential, industrial and military applications. With the ever-increasing development of the renewable energy technology, DC-AC inverters have become one of the most attractive and viable solutions to the power conversion problem. They are extensively used and play key roles in various actual applications of power electronics technologies for renewable energy sources [1-3]. They are also used in motor drive [4,5] and DSTATCOM applications [6] as well as in many uninterruptible power supply system applications such as plant facilities and factories, medical equipments and centers in hospitals, airline computer and communication systems in server farms and web hosting sites [7]. One of the important tasks in the design of DC-AC inverters is the control loop implementation which must ensure a system free from any kind of instabilities. However, it is well known that this aim is difficult to be achieved for all values of system parameters and that many undesired nonlinear phenomena can arise in these kinds of indispensable parts of modern and emerging energy systems. These phenomena can significantly jeopardize the system performance and can cause serious consequences on its reliability. 
Therefore, understanding these nonlinear phenomena, their analysis, prediction and control have increasingly become of great concern of many researchers all over the world [8-22]. The major part of the analytical results on subharmonic oscillation in power electronics converters has been achieved for DC-DC converters [23-40]. DC-AC inverters are more difficult to deal with, since their dynamics is governed by two vastly different frequencies, namely the high switching frequency and the low frequency of the output voltage reference sinewave.

For reliable and desirable operation, the stability of the system must be guaranteed for the whole range of its parameters. In [13], the dynamics behavior of an H-bridge under a digital Current Mode Control (CMC) was investigated by using a one dimensional discrete time model. Different dynamical behaviors for the system were revealed by varying the proportional gain of the current controller. In [14] a similar approach was applied and it was demonstrated that different types of bifurcations (instabilities) can take place such as period doubling leading to Subharmonic Oscillation (SO) and border collision bifurcations leading directly to chaotic behavior.

Using the quasi-static approximation, in [15] the slow-scale and fast-scale instabilities in a voltage-mode controlled H-bridge inverter are reported and analyzed using an averaged model and a discrete-time model respectively. It is well known that conventional averaged model cannot predict the fast-scale instability and for that the discrete-time model must be used. A closed form discrete time model was used in [16] to predict both the slow-scale and the fast-scale instabilities in an H-bridge inverter demonstrating that the system may undergo instability phenomenon when the proportional gain of the voltage controller is increased. In an H-bridge digital-controlled grid-connected inverter system, bifurcation behavior was investigated and loss of system stability was shown by increasing the current controller gain [19] and it was shown that in this system only slow scale instability may take place leading to low-frequency oscillation. The same system, but with double edge modulation, has been studied in [9] using an analytical closed-form expression for predicting a period doubling phenomenon.

Single-stage grid-connected DC-AC conversion systems with boosting voltage capability have recently attracted the attention of many researchers. Single-stage structures of inverters not only perform DC-AC conversion but also perform voltage boosting. Moreover, differential inverter topologies seem to prevail in price and size due to the utilization of small passive elements of DC-DC converters hence improving the efficiency. In contrast to the conventional H-bridge inverter, the differential boost inverter is a flexible DC-AC inverter topology providing voltage step-up capability and could be a potential candidate for many DC-AC electrical energy conversion applications such as for power processing stage fuel-cell energy system [41,42], for high quality sine wave generation with a high oscillation frequency [43], for AC-module microinverters in PV systems such as in [44-46] among others.

In stand-alone operation mode, the load is directly supplied by the inverter. Single-phase H-bridge inverters are simple bidirectional converter topologies capable of handling both real and reactive power having their performance evaluated in terms of power quality and stability. Therefore, generating a high quality output voltage with low distortion and good voltage regulation is the main target. Other relevant performance metrics include disturbance rejection, transient response, and insensitivity to load and system parameter variations. These metrics can only be achieved with a design free from any kind of instability.

Since its introduction in [47], many studies have dealt with the control design of the differential boost inverter using different approaches and strategies [44,45,47-49]. The focus in most of the works published about this inverter is on the control design. However, the analysis of its nonlinear behavior has not been addressed in the past. Namely, SO has not been studied in this kind of inverter. Therefore the aim of this paper is to apply the Floquet theory for accurately predicting the onset of $\mathrm{SO}$ in a differential boost inverter. In contrast to existing works on predicting such a complex behavior in DC-AC inverters based mainly on numerical procedures, here both numerical and analytical approaches are combined to provide a comprehensive study of the systems dynamical behavior. 
The prediction of this phenomenon is of high importance from both theoretical and practical points of view because it leads to an increase in the ripple of the currents and voltages and this has a harmful effect on the system performances since the overall losses become more significant. The power quality can also be jeopardized if $\mathrm{SO}$ is more pronounced since it can increase the THD and the current stress on the switches. Therefore, accurate modeling and stability analysis are necessary for exploring the dynamic behavior and predicting the stability boundaries of DC-AC inverters.

The remaining of this paper is organized as follows. In Section 2, the system dealt with in this study is described. In Section 3, the dynamic behavior of the system is explored revealing that the behavior of the system waveforms is phase-dependent. The system is shown to exhibit local instability phenomenon over a specific interval within the main sinusoidal cycle. The onset of the observed bubbling is associated to a SO phenomenon taking place at the fast switching scale. The mathematical modeling is addressed in Section 4 in the continuous-time domain. In order to analyze the observed phenomena in Section 3, Floquet theory is applied to the derived model in Section 5. Thereafter, in Section 6, the stability boundaries in terms of suitable parameters is reported. Finally, in Section 7 the results of the study are summarized.

\section{Differential Boost Inverter under Two-Loop Control}

The system under study in this paper consists of a differential boost inverter which is obtained by connecting two identical DC-DC boost converters in parallel supplied from a common electrical energy source and feeding a floating voltage load connected between the outputs of the two converters $[47,50]$. Its schematic diagram is shown in Figure 1. The current drawn by the input is shared properly between the two boost converters by the action of a CMC scheme using the difference between the two inductor currents, as will be detailed later. For that, two complementary control signals are considered to control the switches of the differential inverter.

Let us denote the two connected converters as Converter 1 with inductor $L_{1}$ and inductor current $i_{1}$ and Converter 2 with inductor $L_{2}$ and inductor current $i_{2}$. Both converters are controlled in a complementary way using CMC via single Pulse-Width Modulation (PWM) scheme so that Converter 2 is phase shifted $2 \pi D$ with respect to Converter 1 at the switching time scale, $D$ being the operating duty cycle. Namely, the difference between $i_{1}$ and $i_{2}$ (scaled by a sensing resistance $r_{s}$ ) is controlled using a conventional peak CMC by comparing the signal $r_{s}\left(i_{1}-i_{2}\right)$ to the signal $r_{s} i_{\text {ref. }}$. A periodic ramp signal $v_{\text {ramp }}$ with amplitude $V_{M}$ and period $T$ is subtracted from $r_{s} i_{\text {ref }}$ for slope compensation. The comparison of the signal $r_{s}\left(i_{1}-i_{2}\right)$ with the signal $r_{s} i_{\text {ref }}-v_{\text {ramp }}$ by using a comparator and a set-reset flip-flop generate the high and low values of the pulses driving the switches as shown in Figure 1 where the block diagram of the inner current control together with the outer voltage control are depicted.

The reference current for the difference between the two inductor currents is provided by an external voltage loop. The activation of the switches $\mathrm{Q}_{1}, \mathrm{Q}_{2}, \mathrm{Q}_{3}$ and $\mathrm{Q}_{4}$ is carried out as follows: the signal $r_{s}\left(i_{1}-i_{2}\right)$ is connected to the non inverting pin of the comparator whereas the signal $r_{s} i_{\text {ref }}-v_{\text {ramp }}$ is applied to the inverting pin. The output of the comparator is applied to the reset input of a set-reset flip-flop and a periodic clock signal is connected to its set input, as shown in Figure 1, in such a way that the switch $Q_{2}$ and $Q_{4}$ are $O N$ at the beginning of each switching cycle and are turned OFF whenever $r_{s}\left(i_{1}-i_{2}\right)=r_{s} i_{\text {ref }}-v_{\text {ramp }}$. The state of the switches $Q_{1}$ and $Q_{3}$ are complementary to the switches $Q_{2}$ and $\mathrm{Q}_{4}$ respectively.

To fulfill the requirements of the underlying electronic application, a DC-AC inverter has to produce a periodic sinewave-shaped output voltage under normal operational conditions. Let $v_{\text {ref }}(t)$ be the voltage reference that can be expressed as $v_{\text {ref }}(t)=V_{\text {ref }} \sin \left(2 \pi f_{g} t\right)=V_{\text {ref }} \sin (\varphi)$, where $\varphi=2 \pi f_{g} t \in(0,2 \pi)$, $V_{\text {ref }}$ is the peak value of the output voltage reference, $\omega_{0}$ its angular frequency and $\varphi$ its phase angle. In practical applications, the switching frequency is much higher than the AC output voltage frequency. 
This condition is met in this paper and it allows the use of quasi-static approximation. The error voltage $v_{\text {ref }}-v_{0}$ is the input signal to the voltage controller of which the task is to make the output voltage of the inverter an AC sinusoidal signal with zero DC component. Therefore, the load connected between the converters outputs will be subjected to an AC sinusoidal voltage with a zero DC component. This control strategy is different from the one used in most of the published works about this inverter topology such as $[44,45,47]$ where the control is performed such that each boost converter generates a DC bias and an AC component. In the low frequency averaged sense, the AC component of each converter is out of phase regarding the other converter. The DC component is the same for both converters.

The voltage controller is conventionally a PI regulator aiming to make the load voltage $v_{o}$ to accurately track the sinewave voltage reference $v_{\text {ref }}$. Its transfer function can be expressed as $H_{\mathrm{pi}}(s)=k_{p}(\tau s+1) /(s \tau)$, where $k_{p}$ is its proportional gain and $\tau$ is its time constant.

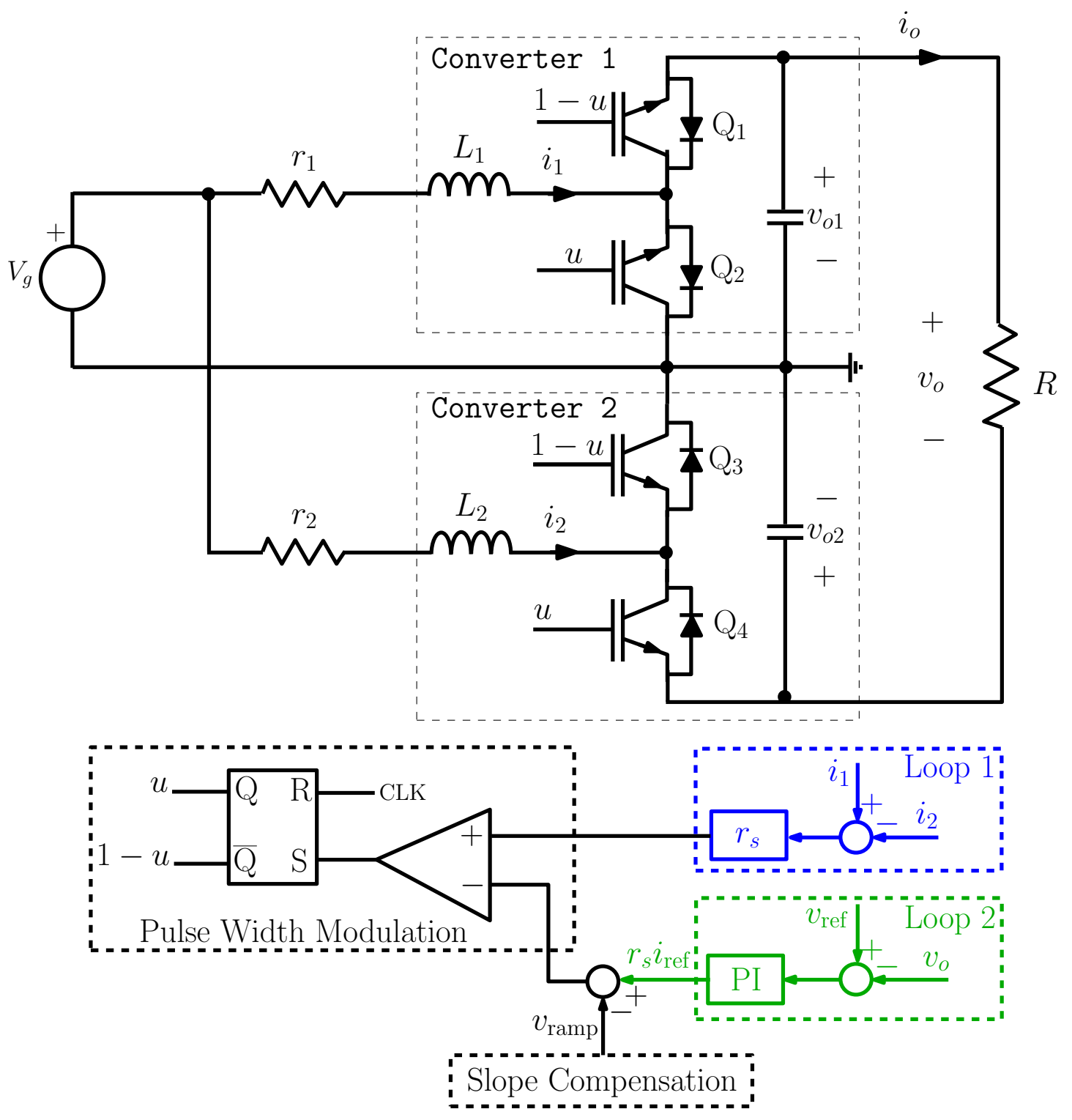

Figure 1. The differential boost inverter under two-loop control. 


\section{Behavior of the Differential Boost Inverter}

The dynamical behavior of the boost inverter is explored in this section with the aim to gain insight on suitable ways of obtaining an appropriate model that can be used for its accurate stability analysis. The system is first studied through simulations using the full-order switched model of the inverter implemented using PSIM(C) software by varying suitable system parameters. The focus is first on system stability in terms of the time varying voltage reference. The fixed parameter values used for the rest of the study are reported in Table 1. Many time-domain waveforms have been computed to get a clear view of the system behavior and only representative results are shown below. The simulation is run for sufficiently long time to allow the system to reach its steady-state. The data obtained during time transient within the startup phase and during the transient regime of the regulation phase are fully eliminated. Only the last two cycles of the output voltage reference are plotted.

Table 1. The used parameters for the DC-AC differential boost inverter.

\begin{tabular}{ll}
\hline Parameter & Value \\
\hline Inductance $L_{1}=L_{2}$ & $100 \mu \mathrm{H}$ \\
Resistance $r_{1}=r_{2}$ & $0.1 \Omega$ \\
Capacitance $C_{1}=C_{2}$ & $22 \mu \mathrm{F}$ \\
Input voltage $v_{g}$ & $200 \mathrm{~V}$ \\
Load resistance $R$ & $100 \Omega$ \\
Time constant of the voltage controller $\tau$ & $1 \mathrm{~ms}$ \\
PWM switching frequency $f_{s}$ & $100 \mathrm{kHz}$ \\
RMS value of the reference voltage $v_{\text {ref }}$ & $230 \mathrm{~V}$ \\
Frequency of the reference voltage $v_{\text {ref }}$ & $50 \mathrm{~Hz}$ \\
Current sensor gain $r_{s}$ & $0.1 \Omega$ \\
\hline
\end{tabular}

Figure 2 shows the system waveforms when the system is stable. The figure shows the time-domain waveforms of the reference voltage $v_{\text {ref }}$ and the output voltage $v_{0}$, the capacitor voltages $v_{01}$ and $v_{02}$, the inductor currents $i_{1}$ and $i_{2}$ and the control signal $r_{s}\left(i_{1}-i_{2}\right)$ and the signal $r_{s} i_{\text {ref }}-v_{\text {ramp. It is worth }}$ noting that the output voltage cannot be distinguished from its reference signal $v_{\text {ref }}$ due to the practically zero amplitude and phase errors. Note also that the state variables and the control signal oscillate at two main frequencies, the switching frequency $(100 \mathrm{kHz})$ and the reference voltage frequency $(50 \mathrm{~Hz})$. From a practical point of view, the output voltage is characterized by a low value of THD as required in any application.

As parameters are varied, the state variables undergo a sudden distortion by exhibiting $\mathrm{SO}$ at the fast switching scale as shown in Figure 3 for $k_{p}=0.4$. This phenomenon takes place when the proportional gain $k_{p}$ gradually increases and reaches a critical value close to 0.22 . As shown in Figure 3, it can be observed that the inductor currents $i_{1}$ and $i_{2}$ exhibit SO leading to disrupting bubbling phenomenon of the waveforms. In particular, when $k_{p} \approx 0.22$, the fast-scale instability develops in all the state variables but it is more visible and pronounced in the inductor current waveforms $i_{1}$ and $i_{2}$ and their combination $r_{s}\left(i_{1}-i_{2}\right)$. As stated before, such behavior manifests itself as a period-doubling phenomenon at the fast switching scale $[9,51]$. It can also be noticed in Figure 4 that the phenomenon already becomes visible in the capacitor voltages and the output voltage hence it can deteriorate the performance of the inverter and therefore its prediction is an important task from a practical point of view. 

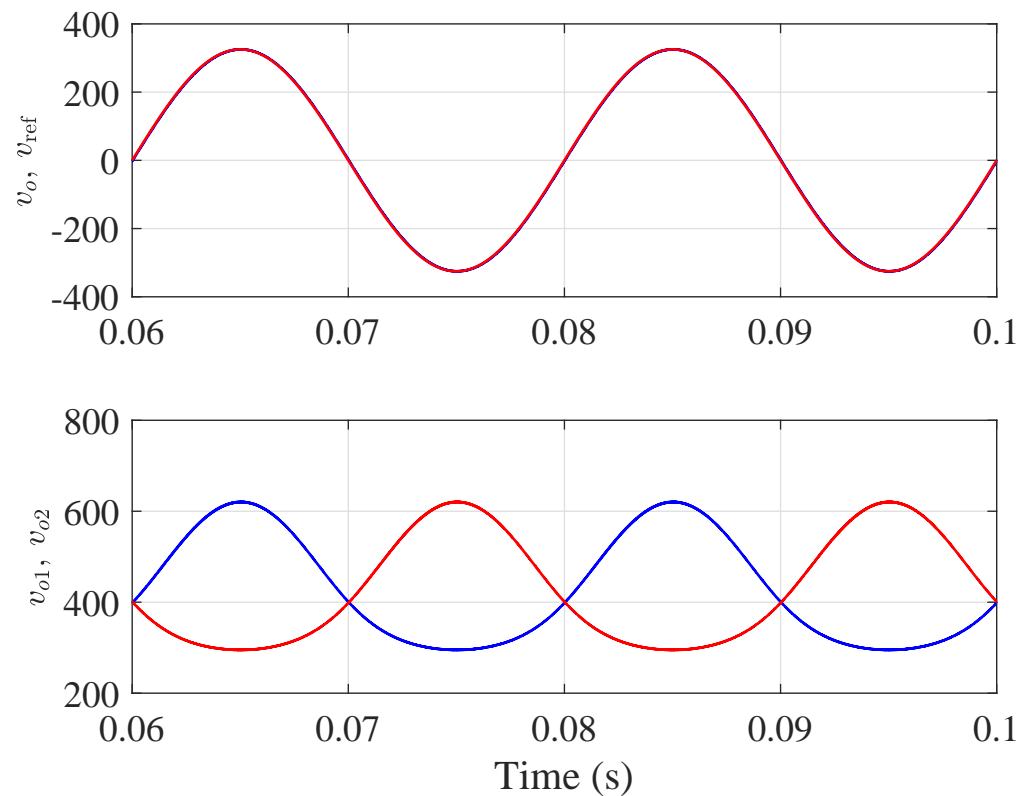

(a)
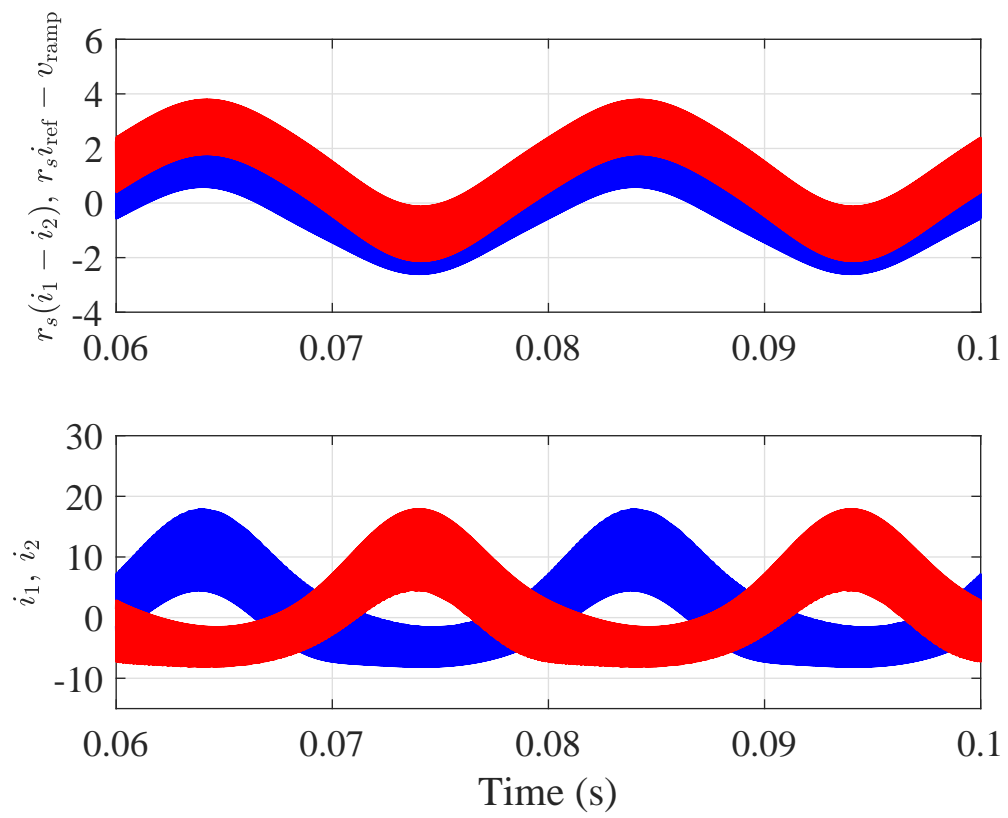

(b)

Figure 2. Steady-state response of boost inverter with $k_{p}=0.2$ and $V_{M}=2 \mathrm{~V}$. (a) Capacitor voltages $v_{01}$ and $v_{02}$, output and reference voltages $v_{0}$ and $v_{\text {ref }}$. (b) Inductor currents $i_{1}$ and $i_{2}$ and control signals $r_{s}\left(i_{1}-i_{2}\right)$ and $r_{s} i_{\text {ref }}-v_{\text {ramp }}$. For each subplot, traces correspond to the shown voltages in [V] and currents in $[\mathrm{A}]$. 

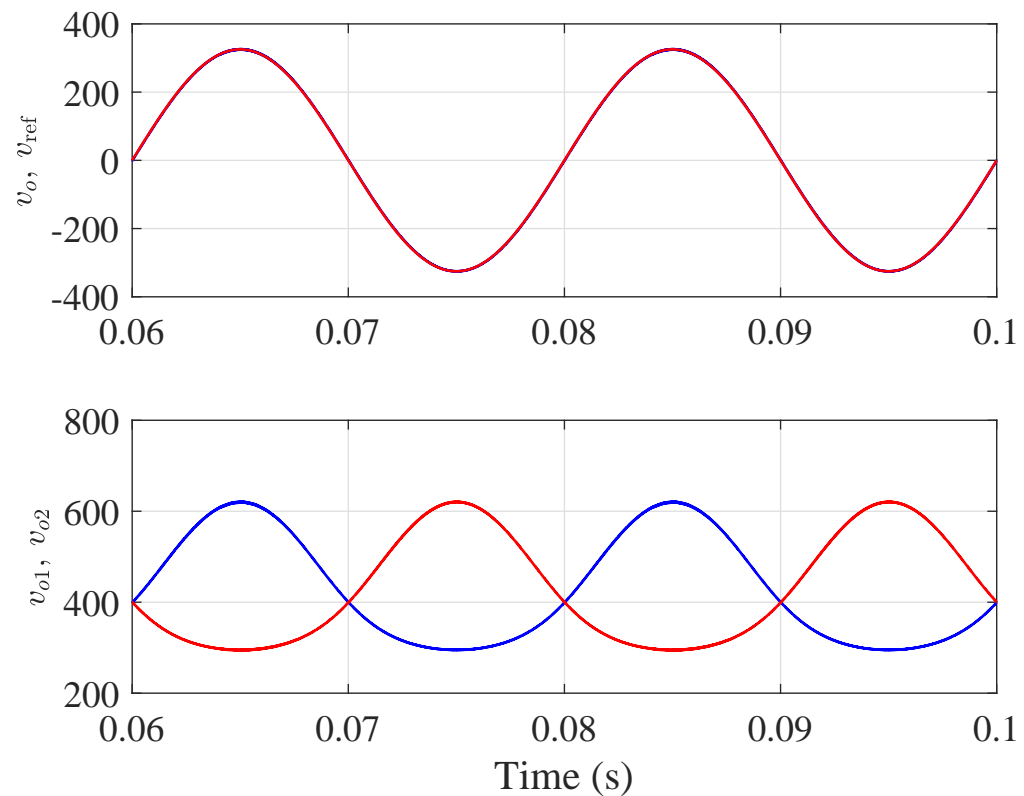

(a)
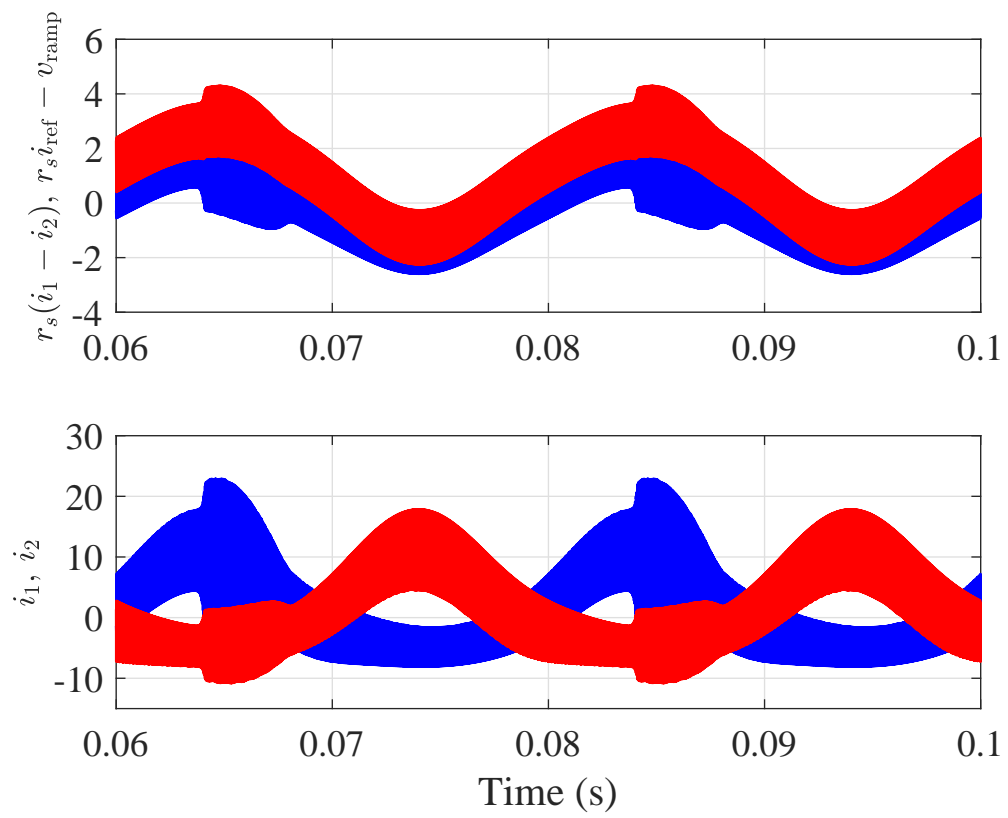

(b)

Figure 3. Steady-state response of boost inverter with $k_{p}=0.4$ and $V_{M}=2 \mathrm{~V}$. (a) Capacitor voltages $v_{01}$ and $v_{02}$, output and reference voltages $v_{0}$ and $v_{\text {ref }}$. (b) Inductor currents $i_{1}$ and $i_{2}$ and control signals $r_{s}\left(i_{1}-i_{2}\right)$ and $r_{s} i_{\text {ref }}-v_{\text {ramp }}$. For each subplot, traces correspond to the shown voltages in [V] and currents in $[\mathrm{A}]$. 

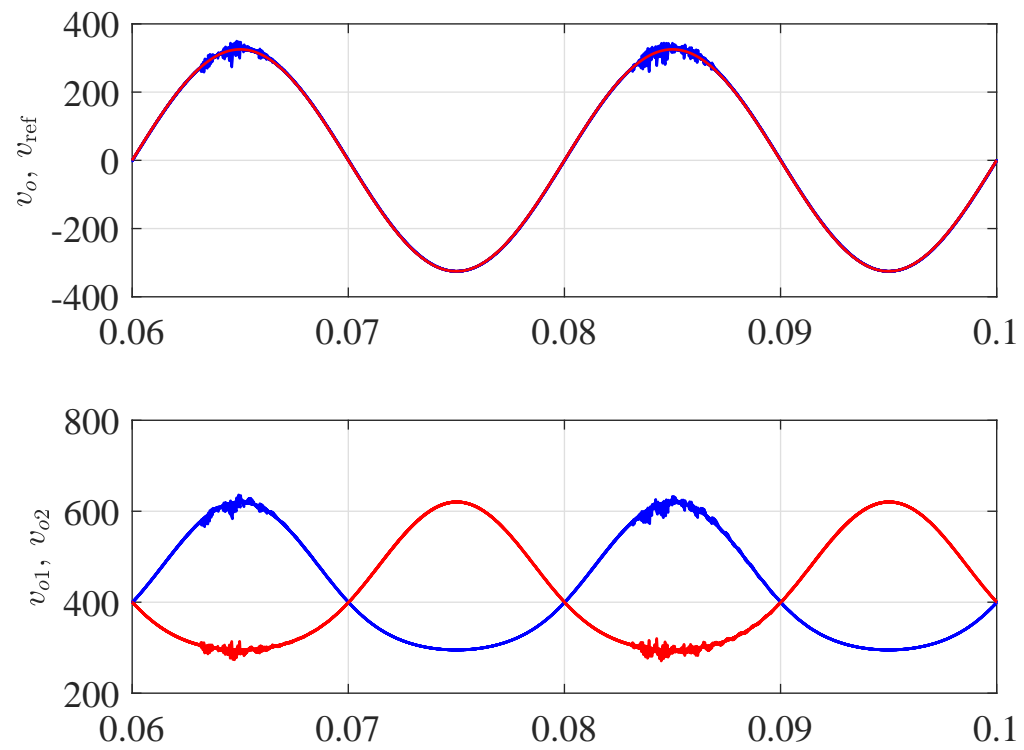

Time (s)

(a)
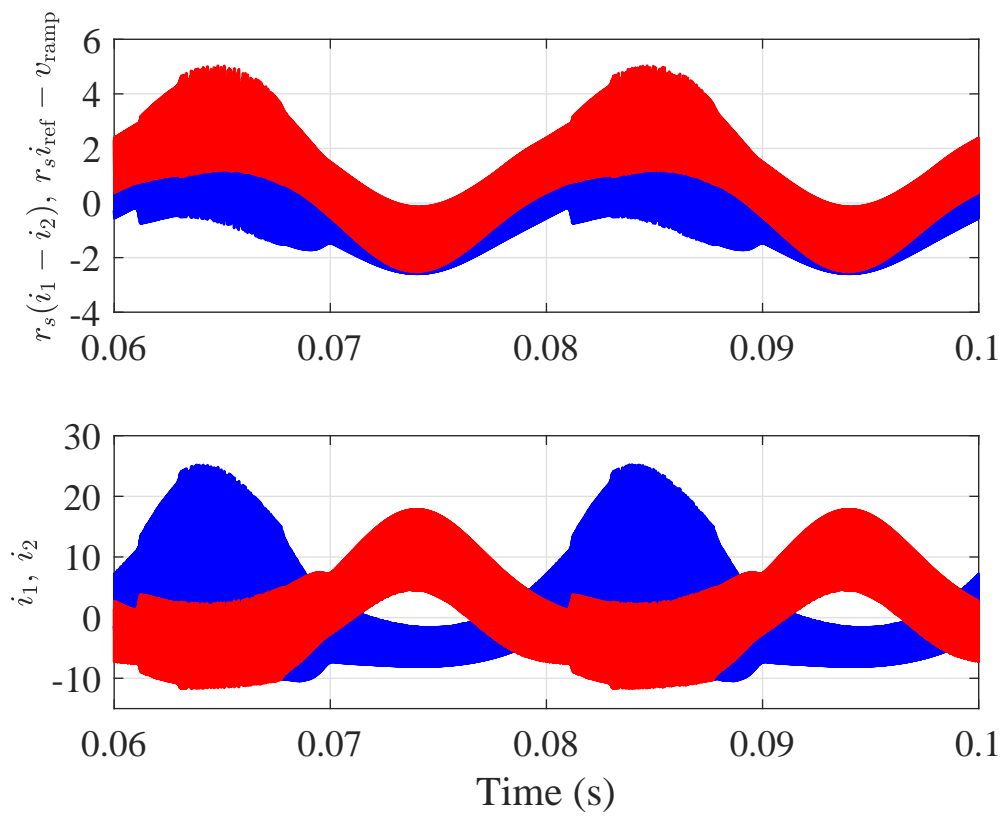

(b)

Figure 4. Steady-state response of boost inverter with $k_{p}=0.8$ and $V_{M}=2 \mathrm{~V}$. (a) Capacitor voltages $v_{01}$ and $v_{02}$, output and reference voltages $v_{0}$ and $v_{\text {ref }}$. (b) Inductor currents $i_{1}$ and $i_{2}$ and control signals $r_{s}\left(i_{1}-i_{2}\right)$ and $r_{s} i_{\text {ref }}-v_{\text {ramp. }}$. For each subplot, traces correspond to the shown voltages in [V] and currents in $[\mathrm{A}]$.

By carefully examining the waveforms, the following statements can be made: 
- By increasing progressively the proportional gain and when this parameter reaches the critical value, SO oscillation starts first occurring in a very limited number of switching cycles during the first half cycle of the sinewave signal eventually in the neighborhood to the quarter of the cycle where the sinewave signal is maximum.

- The number of the switching cycles, during which $\mathrm{SO}$ is exhibited, gets larger and the fast-scale SO is more pronounced when the proportional gain $k_{p}$ is increased.

- At the left and at the right of the maximum values during the same half cycle, one has the same values of quasi-steady-state duty cycles and therefore, theoretically, a perfect symmetry is expected in the critical phase angles at which SO takes place. However, an asymmetry can take place because the slope of the reference sinewave signal at the left of the peak point is positive while it is negative at the right side.

- The SO interval is repetitive from a sinewave cycle to the next one and the study of SO phenomenon can be restricted to one sinewave cycle in terms of the phase angle $\varphi$ as a slowly varying parameter in the range $\varphi \in(0,2 \pi)$.

- Apparently, if SO is avoided for the first half cycle of the sinewave signal, it will also be avoided for the second half cycle. Therefore, the numerical and the analytical studies to be presented later will be restricted to the first half cycle of the sinewave signal for $\varphi \in(0, \pi)$, i.e., only within the duty cycle range $D \in(0.5,1)$.

A powerful tool for clearly illustrating the SO phenomenon is by using the sampled waveforms. In order to clearly appreciate the change in the behavior of the system, sampled steady-state values of the state variables at time instants $t=n T(n \in \mathbb{N})$ are obtained. Therefore, the state variables are sampled at every clock instant and then plotted in the time domain. A priori, any one of the state variables can be used for illustrating the behavior of the system. However, as observed in the previous time domain numerical simulations, $\mathrm{SO}$ is more pronounced in some state variables than others. An interesting and naturally sampled variable for which SO is well noticed is the duty cycle of the binary signal $u$.

Figure 5 shows the waveforms of the duty cycle $d(n T)(n \in \mathbb{N})$ during one complete sinewave cycle for four different values of the proportional gain $k_{p}$. The duty cycle waveforms are plotted in terms of the phase angle within the interval $(0,2 \pi)$. For $k_{p}=0.2$, the system exhibits a stable periodic regime in steady-state, the duty cycle does not present any disruption and its samples represent a clean and smooth waveform. When the SO regime starts taking place, one gets a different picture. For instance, for $k_{p}=0.4$, it can be clearly seen that there is a certain phase interval within the first half of the sinewave cycle during which the duty cycle waveforms is disrupted. Namely, within the phase interval defined by two critical phase angles, two different branches of duty cycle values appear instead of one a kind of bubble emerges [18]. It can be observed that the onset of bubbling phenomenon depicted in Figure 5 is gradual. First, for a relatively small value of the parameter $k_{p}$, the cycle is smooth, then, for increasing $k_{p}$, it becomes disrupted in a small phase interval. Thereafter, as $k_{p}$ is further increased, the interval $\left(\varphi_{1}, \varphi_{2}\right)$ of $\varphi$ during which SO takes place grows up as can be seen in Figure 5. If the proportional gain is further increased, this interval gets wider and the phenomenon usually spreads through the whole line cycle. Figure 5 also shows that successive period doubling inside the SO interval may also take place in the first half cycle where the voltage reference is positive, i.e., when $D>0.5$. When $k_{p}$ becomes even larger, the bubbles start appearing even in the second half cycle of voltage reference where $D<0.5$. Therefore, even for $D<0.5$, the voltage loop may have a destabilizing effect since when the proportional gain $k_{p}$ is increased beyond a critical value $k_{p} \approx 0.8$, SO and the associated bubbling starts appearing for $D<0.5$ and even in the presence of slope compensation. Therefore, the ramp slope needed for eliminating SO is larger than the one obtained when ignoring the effect of the voltage loop. This destabilizing effect of the voltage loop is similar to the one reported in [27] for the buck converter and in [24] for the boost 
converter. Similar behaviors have been obtained when other parameters such as the input voltage $V_{g}$ or the inductance $L$ are varied.

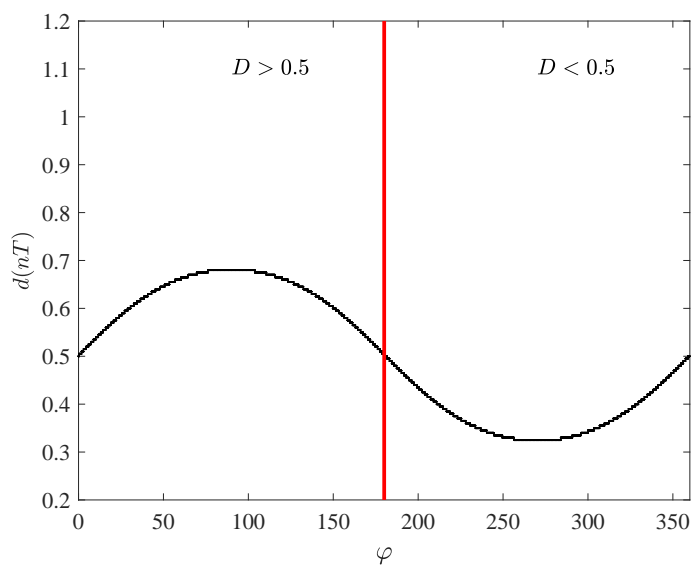

(a) $k_{p}=0.2$.

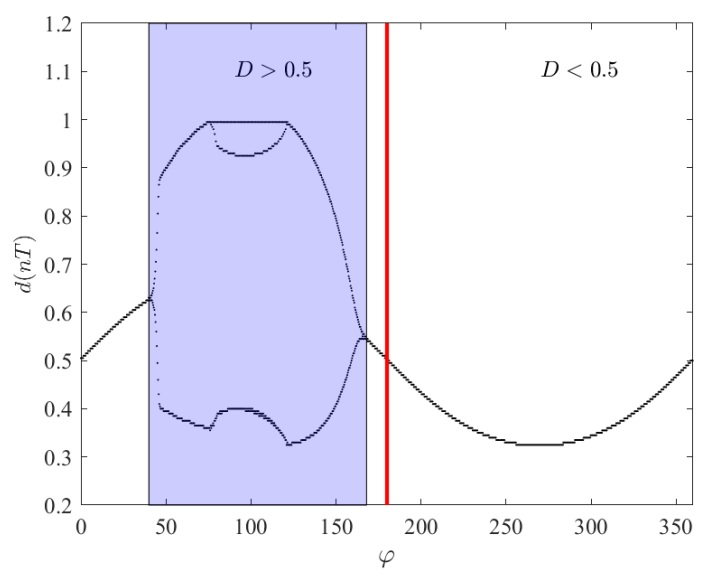

(c) $k_{p}=0.6$.

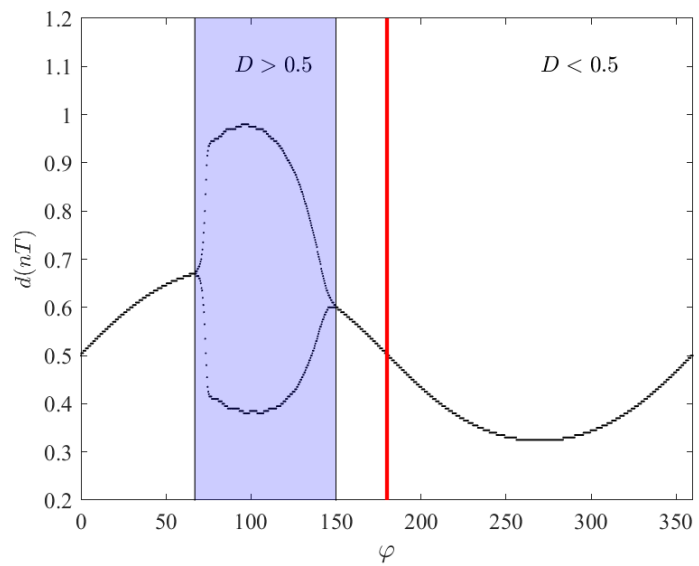

(b) $k_{p}=0.4$.

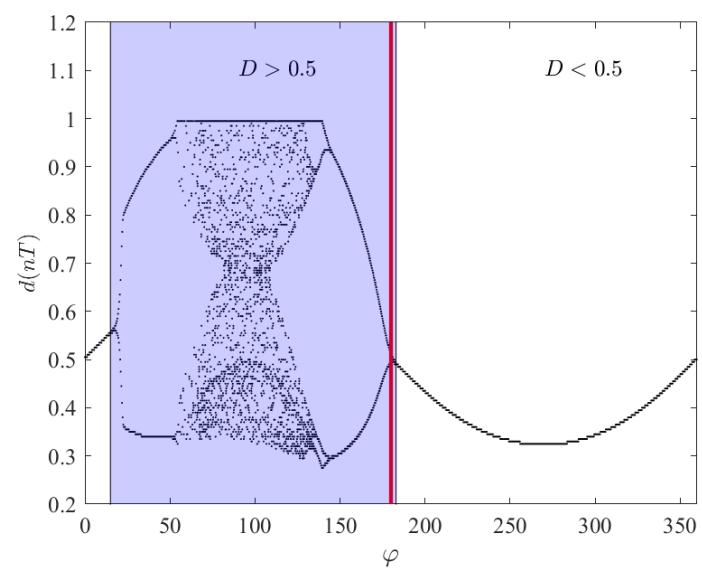

(d) $k_{p}=0.8$.

Figure 5. Waveforms of the duty cycle $d(n T)$ at steady-state operation in terms of the phase angle in $\left[{ }^{\circ}\right]$ for different values of $k_{p}$ and for $V_{M}=2 \mathrm{~V}$.

\section{Continuous-Time Modeling of the Differential Boost Inverter}

\subsection{Quasi-Steady-State Analysis}

From the simulation results presented in the previous section, it has been observed that SO takes place when suitable parameters are varied. One of the widespread tools to analyze and to investigate this kind of nonlinear behavior is Floquet theory [52,53]. Considering the switched model of the system, one can identify possible periodic orbits, their stability as well as several other important aspects of the 
dynamical behavior. To apply this theory, the mathematical model is first derived. By applying KVL and $\mathrm{KCL}$, the switched model of the differential boost inverter can be expressed as follows

$$
\begin{aligned}
\frac{\mathrm{d} i_{1}}{\mathrm{~d} t} & =\frac{1}{L_{1}}\left(V_{g}-v_{o 2}(1-u)\right)-\frac{r_{1}}{L_{1}} i_{1}, \\
\frac{\mathrm{d} i_{2}}{\mathrm{~d} t} & =\frac{1}{L_{2}}\left(V_{g}-u v_{o 2}\right)-\frac{r_{2}}{L_{2}} i_{2}, \\
\frac{\mathrm{d} v_{o 1}}{\mathrm{~d} t} & =\frac{1}{C_{1}}\left((1-u) i_{1}+\frac{v_{o 1}-v_{o 2}}{R}\right), \\
\frac{\mathrm{d} v_{o 2}}{\mathrm{~d} t} & =\frac{1}{C_{2}}\left(u i_{2}-\frac{v_{o 1}-v_{o 2}}{R}\right),
\end{aligned}
$$

where $L_{1}$ and $L_{2}$ are the inductance of the inductors of the differential boost inverter with stray resistances $r_{1}$ and $r_{2}$ respectively, $C_{1}$ and $C_{2}$ are the capacitances of their capacitors. $V_{g}$ is the DC input voltage and $R$ is the AC load resistance. All other parameters appearing in (1)-(4) are shown in Figure 1. The quasi-steady-state average values of the state variables are related to the quasi-steady-state duty cycle $D$ by the following expressions:

$$
\begin{aligned}
I_{1} & =\frac{V_{g}(2 D-1)}{R D(1-D)^{2}}, \quad I_{2}=-\frac{V_{g}(2 D-1)}{R D^{2}(1-D)} \\
V_{o 1} & =\frac{V_{g}}{1-D^{\prime}}, \quad V_{o 2}=\frac{V_{g}}{D}
\end{aligned}
$$

These expressions have been obtained by using the averaged model of the inverter within a switching period. Using (6) and the fact that $v_{0}=v_{01}-v_{02}$, the voltage gain of the differential boost inverter can be expressed as follows

$$
M(D):=\frac{v_{\text {ref }}}{V_{g}}=\frac{2 D-1}{D(1-D)}
$$

The inverter gain $M(D)$ reaches its maximum value $M_{\max }=V_{\text {ref }} / V_{g}$ when the voltage reference $v_{\text {ref }}$ reaches its peak value $V_{\text {ref }}$. From the expression of $M(D)$, the steady-state value of the duty cycle can be derived and this can be expressed as follows

$$
D(t)= \begin{cases}\frac{1}{2}-\frac{V_{g}}{v_{\text {ref }}}+\frac{\sqrt{4 V_{g}^{2}+v_{\text {ref }}^{2}}}{2 v_{\text {ref }}} & \text { if } v_{\text {ref }}(t)>0, \\ \frac{1}{2}-\frac{V_{g}}{v_{\text {ref }}}-\frac{\sqrt{4 V_{g}^{2}+v_{\text {ref }}^{2}}}{2 v_{\text {ref }}} & \text { if } v_{\text {ref }}(t)<0 .\end{cases}
$$

In terms of the phase angle $\varphi$, the quasi-steady-state duty cycle can be expressed as follows

$$
D(\varphi)= \begin{cases}\frac{1}{2}-\frac{1}{M_{\max } \sin (\varphi)}+\sqrt{M_{\max }+\frac{1}{4}} & \text { if } \varphi \in(0, \pi), \\ \frac{1}{2}-\frac{1}{M_{\max } \sin (\varphi)}-\sqrt{M_{\max }+\frac{1}{4}} & \text { if } \varphi \in(\pi, 2 \pi) .\end{cases}
$$

\subsection{The State-Space Switched Model}

Let $\mathbf{x}=\left(i_{1}, i_{2}, v_{01}, v_{o 2},\right)^{\top}$ be the vector of the state variables of the power stage of the inverter. The system can be described by a piecewise linear switched model as follows 


$$
\begin{aligned}
\dot{\mathbf{x}} & =\mathbf{A}_{1} \mathbf{x}+\mathbf{B}_{1} V_{g}, \quad \text { for } u=1, \\
\dot{\mathbf{x}} & =\mathbf{A}_{0} \mathbf{x}+\mathbf{B}_{0} V_{g}, \text { for } u=0, \\
\dot{v}_{i} & =v_{\mathrm{ref}}-\left(v_{o 1}-v_{o 2}\right)=v_{\mathrm{ref}}-\mathbf{C}^{\top} \mathbf{x}
\end{aligned}
$$

where $\mathbf{C}^{\boldsymbol{\top}}=\left(\begin{array}{llll}0 & 0 & 1 & -1\end{array}\right)$ and $v_{i}:=\int\left(v_{\text {ref }}-v_{0}\right) \mathrm{d} t$ is the integral of the error signal $v_{\text {ref }}-v_{0} . \mathbf{A}_{0} \in \mathbb{R}^{4 \times 4}$, $\mathbf{A}_{1} \in \mathbb{R}^{4 \times 4}, \mathbf{B}_{0} \in \mathbb{R}^{4 \times 1}$ and $\mathbf{B}_{1} \in \mathbb{R}^{4 \times 1}$ are the system state matrices presented below. The variable $v_{i}$ was deliberately separated from the rest of state variables to avoid matrix singularities appearing in the expressions of the system trajectories and their steady-state values at the switching time instants $[25,26]$. The matrices $\mathbf{A}_{1}, \mathbf{A}_{0}, \mathbf{B}_{1}$ and $\mathbf{B}_{0}$ are as follows:

$$
\begin{aligned}
& \mathbf{A}_{1}=\left(\begin{array}{cccc}
-\frac{r_{1}}{L_{1}} & 0 & 0 & 0 \\
0 & -\frac{r_{2}}{L_{2}} & 0 & -\frac{1}{L_{2}} \\
0 & 0 & -\frac{1}{R C_{1}} & \frac{1}{R C_{1}} \\
0 & \frac{1}{C_{2}} & \frac{1}{R C_{2}} & -\frac{1}{R C_{2}}
\end{array}\right), \quad \mathbf{B}_{1}=\left(\begin{array}{c}
\frac{1}{L_{1}} \\
\frac{1}{L_{2}} \\
0 \\
0
\end{array}\right) \\
& \mathbf{A}_{0}=\left(\begin{array}{cccc}
-\frac{r_{1}}{L_{1}} & 0 & -\frac{1}{L_{1}} & 0 \\
0 & -\frac{r_{2}}{L_{2}} & 0 & 0 \\
\frac{1}{C_{1}} & 0 & -\frac{1}{R C_{1}} & \frac{1}{R C_{1}} \\
0 & 0 & \frac{1}{R C_{2}} & -\frac{1}{R C_{2}}
\end{array}\right), \mathbf{B}_{0}=\left(\begin{array}{c}
\frac{1}{L_{1}} \\
\frac{1}{L_{2}} \\
0 \\
0
\end{array}\right)
\end{aligned}
$$

The output of the voltage PI voltage controller providing the current reference for the control signal $r_{s}\left(i_{1}-i_{2}\right)$ can be expressed as follows

$$
r_{s} i_{\text {ref }}=k_{p}\left(v_{\text {ref }}-\mathbf{C}^{\top} \mathbf{x}\right)+W_{i} v_{i}
$$

Therefore, the switching condition when the signal $r_{s}\left(i_{1}-i_{2}\right)$ reaches its peak value $r_{s} i_{\text {ref }}-v_{\text {ramp }}$ within a switching cycle is given by

$$
k_{p}\left(v_{\text {ref }}-\mathbf{C}^{\top} \mathbf{x}\right)+W_{i} v_{i}-\left(v_{\text {ramp }}(t)+r_{s}\left(i_{1}-i_{2}\right)\right)=0,
$$

which can be expressed in the following form

$$
k_{p} v_{\text {ref }}+\mathbf{K x}(t)+W_{i} v_{i}(t)-v_{\text {ramp }}(t)=0,
$$

where $\mathbf{K}=\left(\begin{array}{llll}-r_{s} & r_{s} & -k_{p} & k_{p}\end{array}\right)$ is the vector of feedback coefficients.

\section{Accurate Stability Analysis Using Floquet Theory}

The differential equations describing the dynamics of switching converters are time periodic with the switching period $T$ determining the periodicity of solutions at the fast switching scale. DC-AC inverters are also time periodic with the switching period $T$ and the voltage reference period $T_{g}=1 / f_{g}$. For such time periodic systems Floquet theory can be used to study the stability of periodic orbits [53]. Here, this theory 
will be applied using a quasi-static approximation treating the DC-AC inverter as a DC-DC converter with a slowly varying reference voltage and duty cycle. With this approximation, the reference voltage $v_{\text {ref }}$ is considered constant within a switching cycle.

Floquet theory has been widely used in the analysis of stability of dynamical systems [53] in general and switching converters in particular [38-40]. For DC-DC converters, the stability dynamics at the fast switching cycle can be accurately predicted by analyzing the stability of the fixed points of the Poincaré map of the system using its Jacobian matrix or using Floquet theory combined with Filippov method which leads to the same results as the Poincaré map [38]. The main tool for studying the stability of periodic orbits using Floquet theory is the principal fundamental matrix or the monodromy matrix $\mathbf{M}$. This matrix plays a key role in the accurate stability analysis of switching systems $[38-40,53]$. The monodromy matrix is such that the dynamics in the vicinity of a quasi-static periodic orbit can be expressed as follows

$$
\hat{\mathbf{x}}(t+T)=\mathbf{M} \hat{\mathbf{x}}(t) \quad \forall t
$$

where the overhat stands for small signal variations. Its eigenvalues are called the characteristic multipliers or Floquet multipliers and it can be seen that they determine the amount of contraction or expansion near a periodic orbit and hence they determine the stability of these periodic orbits.

Let us start by finding the monodromy matrix $\mathbf{M}$. Let $\mathbf{x}(t) \approx \mathbf{x}(t+T)$ the quasi-steady-state value of the state vector. Let $\mathbf{x}(D T)=(\mathbf{I}-\mathbf{\Phi})^{-1} \mathbf{\Psi} \approx \mathbf{x}(t) \approx \mathbf{x}(D T)$ be the value of $\mathbf{x}(t)$ at time instant $D T$, where $\boldsymbol{\Phi}=\boldsymbol{\Phi}_{1} \boldsymbol{\Phi}_{0}, \boldsymbol{\Phi}_{1}=e^{\mathbf{A}_{1} D T}, \boldsymbol{\Phi}_{0}=e^{\mathbf{A}_{0}(1-D) T}, \boldsymbol{\Psi}_{1}=\left(e^{\mathbf{A}_{1} D T}-\mathbf{I}\right) \mathbf{A}_{1}^{-1} \mathbf{B} V_{g}, \mathbf{\Psi}_{0}=\left(e^{\mathbf{A}_{0}(1-D) T}-\mathbf{I}\right) \mathbf{A}_{0}^{-1} \mathbf{B} V_{g}$, $\mathbf{\Psi}=\boldsymbol{\Phi}_{1} \Psi_{0}+\Psi_{1}$. Let $\mathbf{m}_{1}(\mathbf{x}(t))=\mathbf{A}_{1} \mathbf{x}(t)+\mathbf{B}_{1} V_{g}$ and $\mathbf{m}_{0}(\mathbf{x}(t))=\mathbf{A}_{0} \mathbf{x}(t)+\mathbf{B}_{0} V_{g}$ be the vector fields for $u=1$ and $u=0$ respectively. Let us define the augmented state vector $\mathbf{x}_{a}=\left(i_{1}, i_{2}, v_{o 1}, v_{o 2}, v_{i}\right)^{\top}$. Let $\mathbf{A}_{a 1}$, $\mathbf{A}_{a 0}, \mathbf{B}_{a 1}, \mathbf{B}_{a 0}, \mathbf{w}_{a}$ and $\mathbf{K}_{a}$ be, respectively, the associated augmented state matrices, input vectors, vector of external parameters and vector of feedback coefficients that are expressed as follows

$$
\begin{aligned}
& \mathbf{A}_{a 1}=\left(\begin{array}{ll}
\mathbf{A}_{1} & \mathbf{0} \\
-1 & \mathbf{0}
\end{array}\right), \mathbf{B}_{a 1}=\left(\begin{array}{cc}
\mathbf{B}_{1} & 0 \\
\mathbf{0} & 1
\end{array}\right) \\
& \mathbf{A}_{a 0}=\left(\begin{array}{ll}
\mathbf{A}_{0} & \mathbf{0} \\
-1 & \mathbf{0}
\end{array}\right), \mathbf{B}_{a 0}=\left(\begin{array}{cc}
\mathbf{B}_{0} & 0 \\
\mathbf{0} & 1
\end{array}\right) \\
& \mathbf{K}_{a}=\left(\mathbf{K} W_{i}\right), \mathbf{w}_{a}=\left(\begin{array}{c}
V_{g} \\
v_{\text {ref }}
\end{array}\right)
\end{aligned}
$$

Let us also define the augmented state transition matrices $\boldsymbol{\Phi}_{a 1}=e^{\mathbf{A}_{a 1} D T}$ and $\boldsymbol{\Phi}_{a 0}=e^{\mathbf{A}_{a 0}(1-D) T}$ and the augmented vector fields $\mathbf{m}_{a 1}\left(\mathbf{x}_{a}(t)\right)=\mathbf{A}_{a 1} \mathbf{x}_{a}(t)+\mathbf{B}_{a 1} \mathbf{w}_{a}$ and $\mathbf{m}_{a 0}\left(\mathbf{x}_{a}(t)\right)=\mathbf{A}_{a 0} \mathbf{x}_{a}(t)+\mathbf{B}_{a 0} \mathbf{w}_{a}$. Then, the full-order monodromy matrix can be expressed as follows [38]

$$
\mathbf{M}=\boldsymbol{\Phi}_{a 0} \mathbf{S} \boldsymbol{\Phi}_{a 1}
$$

where $\mathbf{S}$ is the saltation matrix adapted from [38] as follows

$$
\mathbf{S}=\mathbf{I}+\frac{\left(\mathbf{m}_{a 0}\left(\mathbf{x}_{a}(D T)\right)-\mathbf{m}_{a 1}\left(\mathbf{x}_{a}(D T)\right)\right) \mathbf{K}_{a}^{\top}}{W_{i}\left(v_{\mathrm{ref}}-v_{o}(D T)\right)+\mathbf{K}^{\top} \mathbf{m}_{1}(\mathbf{x}(D T))-m_{\mathrm{ramp}}-m_{\mathrm{ref}}} .
$$

where $m_{\text {ramp }}=V_{M} / T$ is the slope of the ramp compensator and $m_{\text {ref }}=k_{p} V_{\text {ref }} 2 \pi f_{g} \cos \left(2 \pi f_{g} D T\right)$ is the slope contributed by the time variation of the sinusoidal voltage reference. The expression of $v_{i}(D T)$, 
the third component of $\mathbf{x}_{a}(D T)$, can be obtained from (17) in steady-state which gives the following expression for $v_{i}(D T)$

$$
v_{i}(D T)=\frac{1}{W_{i}}\left(m_{\mathrm{ramp}} D T-\mathbf{K}^{\top} \mathbf{x}(D T)-k_{p} v_{\mathrm{ref}}\right)
$$

Now that the expression of the monodromy matrix was derived, hereinafter, we will pay special attention to the movement of the Floquet multipliers as the voltage reference $v_{\text {ref }}$ varies quasi-statically. This is equivalent to changing the phase angle $\varphi$ or the quasi-steady-state duty cycle $D$. We will also study the movement of the Floquet multipliers when the proportional gain $k_{p}$ of the controller or the amplitude of the ramp compensator $V_{M}$ are varied. Any crossing from the interior of the unit circle to its exterior indicates a lost of stability of the desired orbit. The system becomes unstable, if at least one root of the Floquet multiplier leaves the unit circle, which is equivalent to an eigenvalue $\mathbf{M}$ leaving the unit circle. Thus, for the stability boundary $|\lambda|=1$ for at least one eigenvalue of $\mathbf{M}$ holds. In particular, if a real characteristic multiplier goes through -1 as it moves out of the unit circle, $\mathrm{SO}$ at the fast switching scale takes place.

To locate the boundary of SO, the Floquet multipliers are obtained. By varying the quasi-steady-state duty cycle $D$, the operating point $\mathbf{x}(D T)$ was first calculated and the monodromy matrix was obtained for two different values of the proportional gain $k_{p}$. At a point where a subharmonic regime emerges, one of the eigenvalues is equal to -1 . Figure 6 shows the Floquet multipliers loci in the complex plane when the quasi-steady-state duty cycle varies. The duty cycle $D$ was varied by varying the voltage reference between 0 and its maximum values giving rise to $D \in(0.5,0.68)$. As it can be observed from Figure $6 a$, for $k_{p}=0.2$ all the eigenvalues remain inside the unit circle for the full considered range of the duty cycle. Then, the gain $k_{p}$ was fixed at $k_{p}=0.24$ then the reference voltage was varied in the same range as before and the results are depicted in Figure $6 \mathrm{~b}$. It can be observed that one of the eigenvalues of the monodromy matrix crosses the unit circle from the point $(-1,0)$ in the complex plane indicating $\mathrm{SO}$ at a certain value of $v_{\text {ref }}$ very close to its maximum value. The critical value of $k_{p}$ at which this starts taking place is $k_{p} \approx 0.22$ which is in a remarkable agreement with the time-domain numerical simulations presented in Section 3.

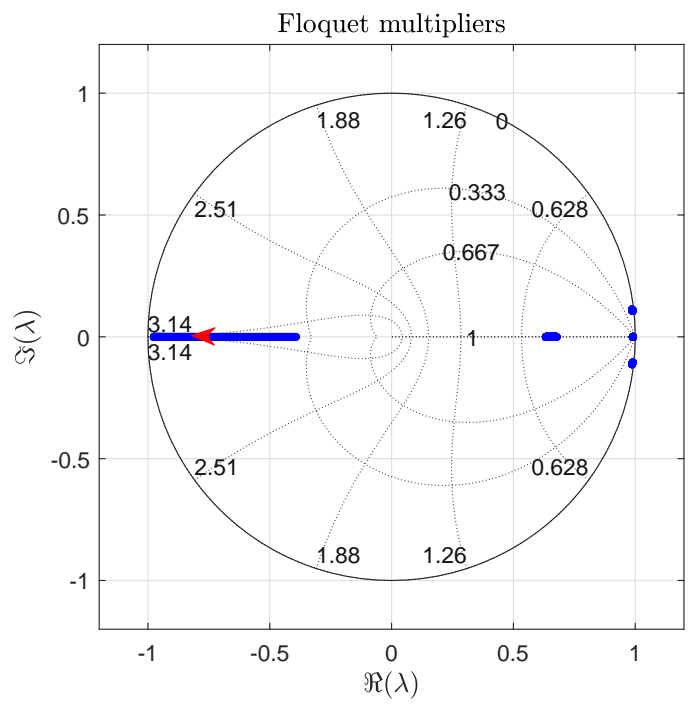

(a) $V_{\text {ref }} \in(0,230 \sqrt{2}) \mathrm{V}(D \in(0.5,0.68))$ and $k_{p}=0.20$.

Figure 6. Cont. 


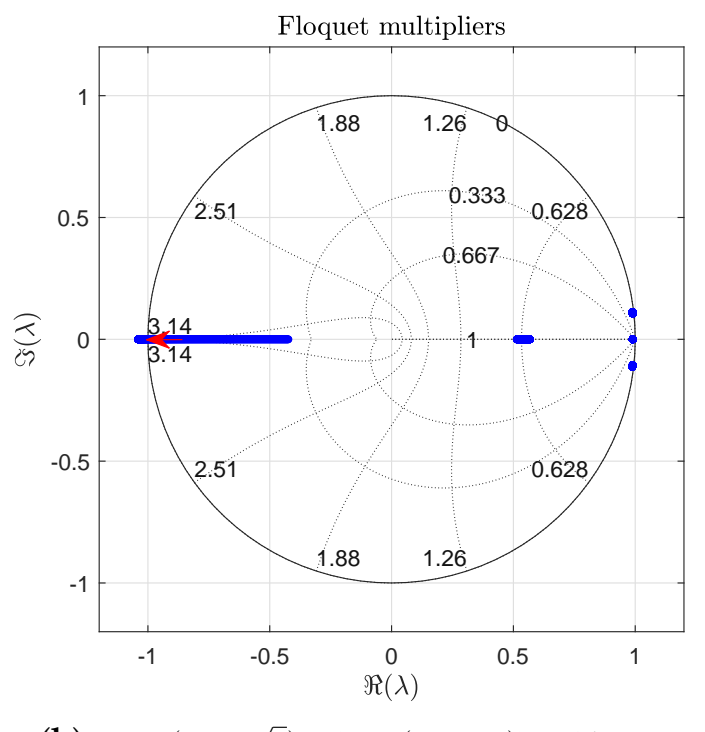

(b) $V_{\text {ref }} \in(0,230 \sqrt{2}) \mathrm{V}(D \in(0.5,0.68))$ and $k_{p}=0.24$.

Figure 6. Floquet multipliers loci by varying the quasi-steady-state duty cycle $D$ for two different values of the proportional gain $k_{p}$.

\section{Stability Boundaries in the Parameter Space}

If $\mathrm{SO}$ boundary is of concern, the expression of the characteristic equation $\operatorname{det}(\mathbf{M}-\lambda \mathbf{I})=0$ can be used by imposing that an eigenvalue $\lambda=-1$ and solving the resulting equation in a suitable projection of the parametric space. Therefore, to determine the boundary of $\mathrm{SO}$, the following equation is solved for a certain system parameter after fixing the other ones

$$
\operatorname{det}(\mathbf{M}+\mathbf{I})=0
$$

The great advantage of using (25) is that only this equation has to be solved without the need of computing all eigenvalues of $\mathbf{M}$ explicitly. Therefore, instead of solving for all eigenvalues of $\mathbf{M}$, only (25) is solved, hence, the saving of computational load is significant when the stability boundary is to be determined.

Figure 7 shows the stability boundary resulted from solving (25) with respect to the proportional gain $k_{p}$ for values of the duty cycle within the operating range $(0.5,0.68)$ and for a value of the ramp compensator amplitude $V_{M}=2 \mathrm{~V}$. Within one sinewave signal one has 2000 switching cycles. Therefore, the plot was generated using 1000 values of the duty cycles and the critical values of $k_{p}$ were registered in terms of $D$. In particular, for $V_{M}=2 \mathrm{~V}$, the critical value of the proportional gain guaranteeing that all the eigenvalues lie inside the unit circle for all values of the operating duty cycle is about 0.2 . This is in perfect agreement with the numerical simulations presented in Section 3. If $V_{M}$ is increased, the critical value of the proportional gain also increases and the stability region gets wider as depicted in Figure 8. In particular, for $V_{M}=3 \mathrm{~V}$, the critical value of the proportional gain is about 0.73 , for $V_{M}=4 \mathrm{~V}$, is about 1.28 and for $V_{M}=5 \mathrm{~V}$, it is about 1.82. Notice that for a fixed switching period $T$, changing the ramp amplitude is equivalent to changing its slope. 


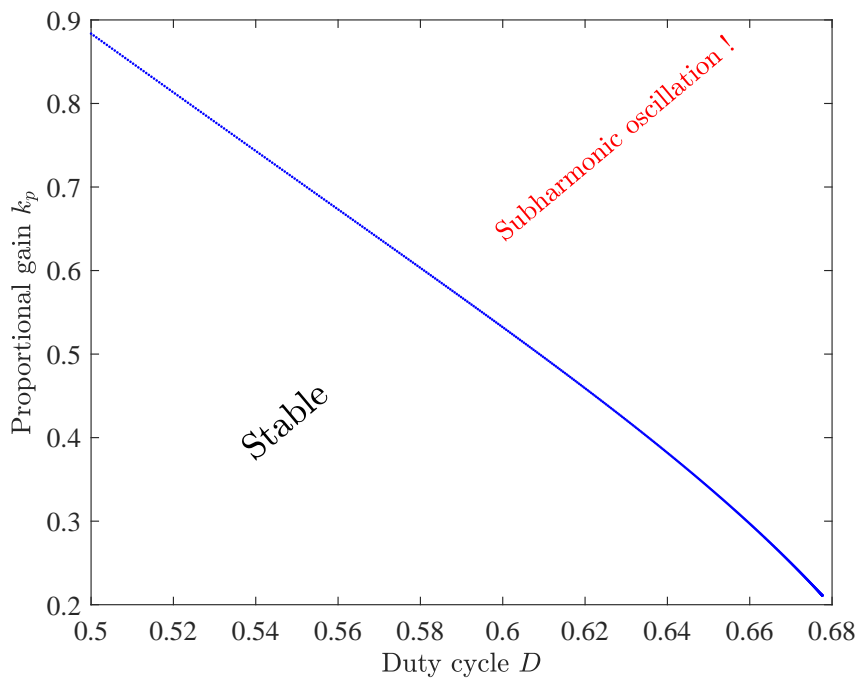

Figure 7. Stability boundaries in terms of the proportional gain $k_{p}$ and the quasi-steady-state duty cycle $D$ and for $V_{M}=2 \mathrm{~V}$.

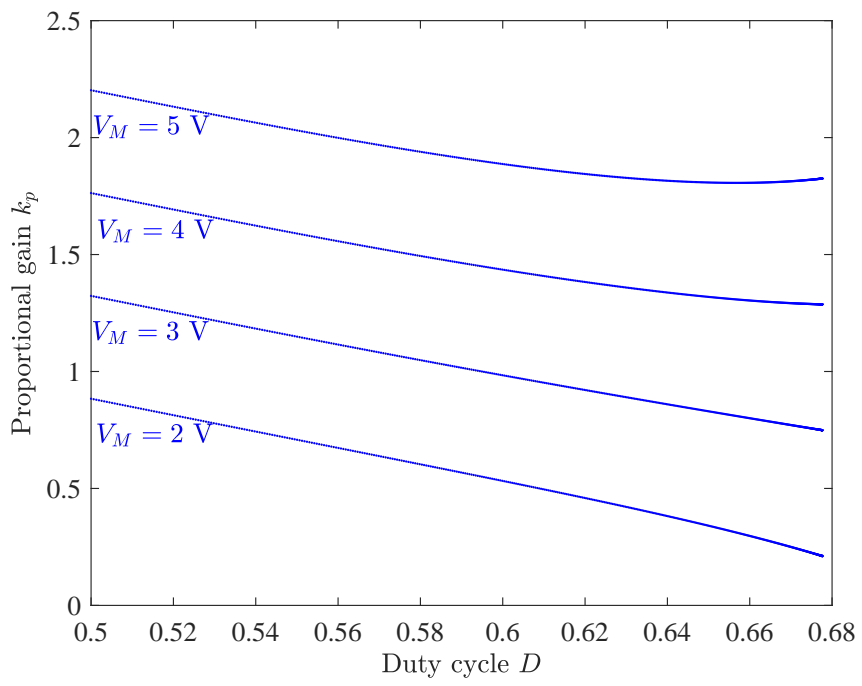

Figure 8. Stability boundaries in terms of the proportional gain $k_{p}$ and the quasi-steady-state duty cycle $D$ for different values of the ramp amplitude $V_{M}$.

As stated previously, in DC-AC inverters, the reference voltage is a time varying sinusoidal signal and accordingly the steady-state quasi-static duty cycle $D$ is given by (8). In such a situation, the phase $\varphi$ is a quasi-static parameter like $D$. Solving (9) in terms of the phase angle $\varphi$, one gets two critical values of the phase angle that can be expressed as follows

$$
\begin{aligned}
\varphi_{1} & =\sin ^{-1}\left(\frac{V_{g}(2 D-1)}{V_{\text {ref }} D(1-D)}\right) \\
\varphi_{2} & =\pi-\sin ^{-1}\left(\frac{V_{g}(2 D-1)}{V_{\text {ref }} D(1-D)}\right)
\end{aligned}
$$


These closed expressions for the critical phase angles at which SO develops explain the observation made in Section 3. In terms of the inverter gain $M(D)$ and its maximum value $M_{\max }$, the expressions of the phase angles are given by

$$
\begin{aligned}
\varphi_{1} & =\sin ^{-1}\left(\frac{M(D)}{M_{\max }}\right) \\
\varphi_{2} & =\pi-\sin ^{-1}\left(\frac{M(D)}{M_{\max }}\right)
\end{aligned}
$$

The stability boundary of the system is plotted in Figure 9, in terms of the proportional gain $k_{p}$ of the voltage controller and the phase angle $\varphi \in(0, \pi)$. Vertical dashed lines in Figure 9 indicate this theoretical critical value for the set of parameter values shown in Table 1 . For each specific union of $\varphi_{1}$ and $\varphi_{2}$ curves, it can be noted that there is a turning point at the left side of the union. The system will be stable at the left of the turning point and will exhibit an SO phenomenon at its right side. For instance, let $k_{p}=0.2$; the system is stable during the entire sinewave cycle as already observed in Figure 5a. When the proportional gain $k_{p}$ is increased beyond its critical value, $\mathrm{SO}$ takes place within a certain phase interval, the length of which is determined by the intersection points between vertical lines corresponding to specific values of $k_{p}$ and the two curves of $\varphi_{1}$ and $\varphi_{2}$. Notice that the length of the SO interval gets larger when the proportional gain increases. For instance, for $k_{p}=0.4$, it is expected from Figure 9 that the system will exhibit SO in the phase interval $\left(\varphi_{1}, \varphi_{2}\right)=\left(46^{\circ}, 134^{\circ}\right)$ which is in close agreement with the numerical simulation depicted in Figure $5 \mathrm{~b}$. For $k_{p}=0.6$, the expected SO interval is $\left(\varphi_{1}, \varphi_{2}\right)=\left(24^{\circ}, 156^{\circ}\right)$ which is in close agreement with Figure $5 \mathrm{c}$ and for $k_{p}=0.8$, the expected SO interval is $\left(\varphi_{1}, \varphi_{2}\right)=\left(7^{\circ}, 173^{\circ}\right)$ which is in close agreement with Figure $5 \mathrm{~d}$.

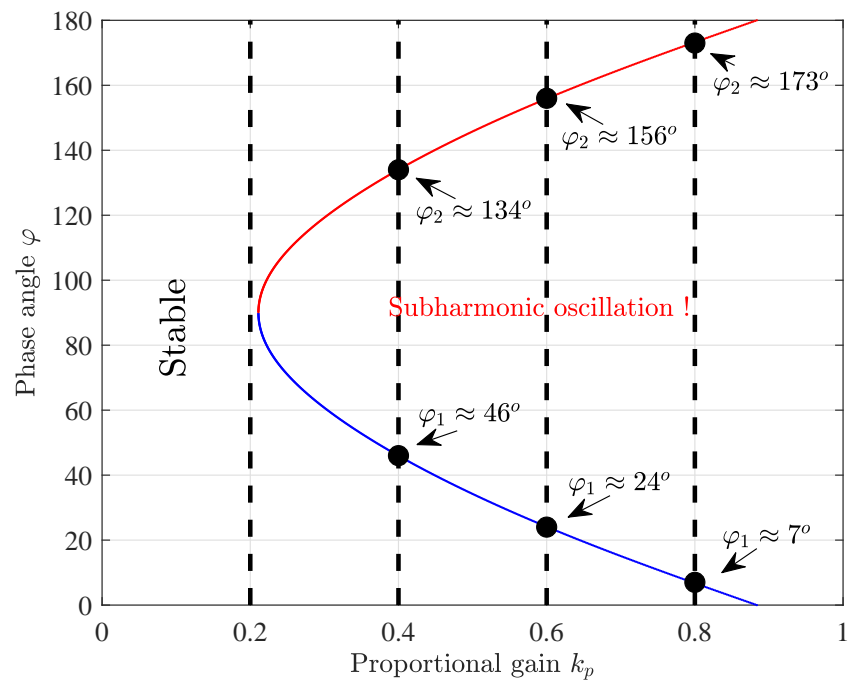

Figure 9. Critical phase angles in $\left[^{\circ}\right]$ defining the SO interval in terms of the proportional gain $k_{p}$.

The estimated values of the critical phase angles from Figure 9 defining the SO interval differ slightly from the numerical simulation result in Figure 5. The discrepancies between the theoretically predicted values in Figure 9 and the ones obtained from numerical simulations depicted in Figure 5 can be attributed to two main factors. The first one is the use of the quasi-static approximation. The second one is the fact that at the point where bubbling develops its amplitude is extremely small making it invisible in 
the scale used for representing the complete waveforms of the duty cycle during one entire sinewave cycle. By zooming close the critical values of $\varphi$, more accurate data can be obtained and discrepancies decrease significantly.

As has been shown in Figure 8, the maximal value of the proportional gain $k_{p}$ guaranteeing stability during the entire the sinewave cycle depends on the ramp amplitude $V_{M}$. Therefore, the critical phase angle curves depicted in Figure 9 are also obtained for different values of $V_{M}$ and the results are depicted in Figure 10. For each ramp amplitude $V_{M}$, a value of the proportional gain $k_{p}$ selected at the left of the corresponding turning point will guarantee no presence of SO during the entire sinewave cycle. Note that as the ramp amplitude $V_{M}$ increases the maximal value allowed for the proportional gain $k_{p}$ also increases.

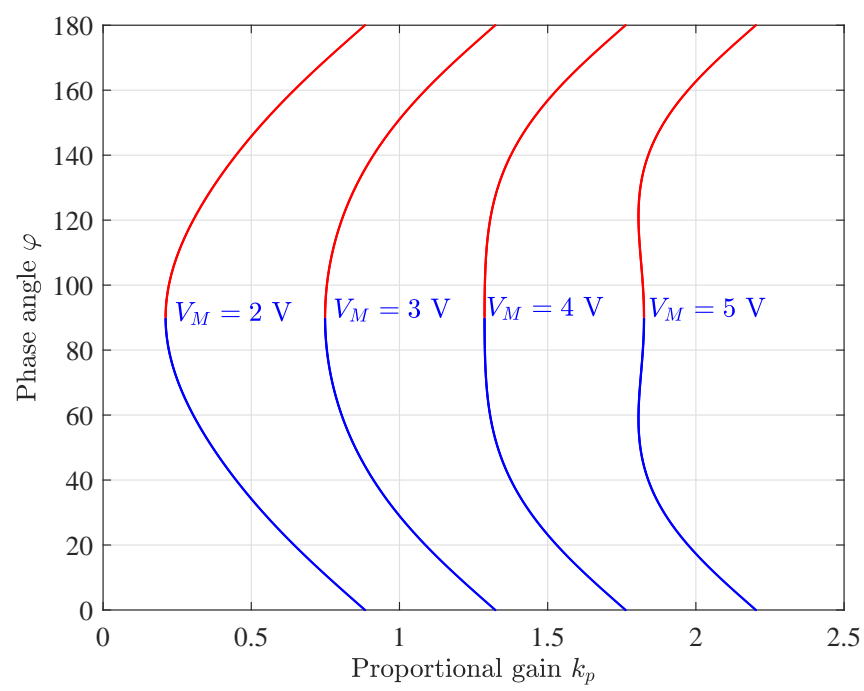

Figure 10. Critical phase angles in $\left[^{\circ}\right]$ defining the SO interval in terms of the proportional gain $k_{p}$ for different values of the ramp amplitude $V_{M}$.

\section{Conclusions}

This paper has focused on the subharmonic oscillation boundary leading to bubbling phenomenon in a single-phase DC-AC differential boost inverter with a linear resistive load. This work has provided a comprehensive study of the system and stability problems of the system were discussed in order to determine stabilizing parameter space. This facilitates convenient selection of parameter values to avoid distortion due to subharmonic oscillation instability in some intervals of the sinewave voltage reference. Therefore, the results are useful for practical design of DC-AC inverters to ensure a stable operation and hence maintain a high power quality and ensuring low and acceptable values of THD. By using time domain waveforms computed from the circuit-level switched model of the system, it was shown that the differential boost inverter could exhibit subharmonic oscillation instabilities at the fast switching scale. Stable and unstable zones of operation, critical parameter values and stability boundaries have been determined. Floquet theory combined with quasi-static approximation has been used resulting in accurately locating the critical values of the system parameters. The theoretical predictions are in perfect agreement with the results obtained from numerical simulations performed on the circuit-level switched model of the inverter. The methodology presented in this study can be applied to other inverter topologies. 
Author Contributions: Conceptualization, A.E.A.; methodology, A.E.A. software, A.E.A.; validation, M.A.-N.; formal analysis, A.E.A.; investigation, A.E.A.; resources, M.A.-N.; data curation, R.H.; writing-original draft preparation, A.E.A.; writing-review and editing, M.A.-N. and M.H.; visualization, M.A.-N., R.H. and M.H.; supervision, M.A.-N.; funding acquisition, M.A.-N. All authors have read and agreed to the published version of the manuscript.

Funding: This work has been sponsored by the Spanish Agencia Estatal de Investigación (AEI) and the Fondo Europeo de Desarrollo Regional (FEDER) under grants DPI2017-84572-C2-1-R. A. El Aroudi and M. Al-Numay acknowledge financial support from the Researchers Supporting Project number (RSP-2020/150), King Saud University, Riyadh, Saudi Arabia.

Conflicts of Interest: The authors declare no conflict of interest.

\section{Abbreviations}

The following abbreviations are used in this manuscript:

$\begin{array}{ll}\text { AC } & \text { Alternate Current } \\ \text { DC } & \text { Direct Current } \\ \text { CMC } & \text { Current Mode Control } \\ \text { PWM } & \text { Pulse Width Modulation } \\ \text { SO } & \text { Subharmonic Oscillation }\end{array}$

\section{References}

1. Yang, C.-Y.; Hsieh, C.-Y.; Feng, F.-K.; Chen, K.-H. Highly efficient analog maximum power point tracking (AMPPT) in a photovoltaic system. IEEE Trans. Circuits Syst. Reg. Pap. 2012, 59, 1546-1556. [CrossRef]

2. Wai, R.-J.; Wang, W.-H. Grid-connected photovoltaic generation system. IEEE Trans. Circuits Syst. Reg. Pap. 2008, 55, 953-964.

3. Fang, Y.; Zhu, Y.; Fei, J. Adaptive intelligent sliding mode control of a photovoltaic Grid-connected inverter. Appl. Sci. 2018, 8, 1756. [CrossRef]

4. Sakharuk, T.A.; Stankovic, A.M.; Tadmor, G.; Eirea, G. Modeling of PWM inverter-supplied AC drives at low switching frequencies. IEEE Trans. Circuits Syst. Fundam. Theory Appl. 2002, 49, 621-631. [CrossRef]

5. Lee, K.; Ha, J.-I. Single-phase inverter drive for interior permanent magnet machines. IEEE Trans. Power Electron. 2017, 32, 1355-1366. [CrossRef]

6. Gupta, R.; Ghosh, A. Frequency-domain characterization of sliding mode control of an inverter used in DSTATCOM application. IEEE Trans. Circuits Syst. Reg. Pap. 2006, 53, 662-676. [CrossRef]

7. Kawamura, A.; Chuarayapratip, R.; Haneyoshi, T. Deadbeat control of PWM inverter with modified pulse patterns for uninterruptible power supply. IEEE Trans. Ind. Electron. 1988, 35, 295-300. [CrossRef]

8. Bandyopadhyay, A.; Howrah, S.; Mandal, K. Design-oriented dynamical analysis of single-phase H-bridge inverter. In Proceedings of the 2020 IEEE International Conference on Power Electronics, Smart Grid and Renewable Energy (PESGRE2020), Cochin, India, 2-4 January 2020.

9. El Aroudi, A.; Al-Numay, M.S.; Lu, W.G.; Bosque-Moncusi, J.M.; Iu, H.H. A combined analytical-numerical methodology for predicting subharmonic oscillation in H-bridge inverters under double edge modulation. IEEE Trans. Circuits Syst. Reg. Pap. 2018, 65, 2341-2351. [CrossRef]

10. Avrutin, V.; Mosekilde, E.; Zhusubaliyev, Z.T.; Gardini, L. Onset of chaos in a single-phase power electronic inverter. Chaos Interdiscip. J. Nonlinear Sci. 2015, 25, 43114. [CrossRef]

11. El Aroudi A.; Orabi A.; Martinez-Salamero, L. A representiative discrete-time model for uncovering slow and fast scale instabilities in boost power factor correction AC-DC pre-regulators. Int. J. Bifurc. Chaos 2008, 18, 3073-3092. [CrossRef]

12. Shankar, D.P.; Govindarajan, U.; Karunakaran, K. Period-bubbling and mode-locking instabilities in a full-bridge dc-ac buck inverter. IET Power Electron. 2013, 6, 1956-1970. [CrossRef]

13. Robert, B.; Robert, C. Border collision bifurcations in a one-dimensional piecewise smooth map for a PWM current-programmed h-bridge inverter. Int. J. Control. 2002, 75, 1356-1367. [CrossRef] 
14. Asahara, H.; Kousaka, T. Bifurcation analysis in a PWM current controlled H-bridge inverter. Int. J. Bifurc. Chaos 2011, 21, 985-996. [CrossRef]

15. Li, M.; Dai, D.; Ma, X. Slow-scale and fast-scale instabilities in voltage-mode controlled full-bridge inverter. Circuits Syst. Signal Process. 2008, 27, 811-831. [CrossRef]

16. El Aroudi, A.; Rodriguez, E.; Orabi, M.; Alarcon, E. Modeling of switching frequency instabilities in buck-based dc-ac H-bridge inverters. Int. J. Circuit Theory Appl. 2011, 39, 175-193. [CrossRef]

17. Zhusubaliyev, Z.T.; Mosekilde, E.; Andriyanov, A.I.; Shein, V.V. Phase synchronized quasiperiodicity in power electronic inverter systems. Phys. Nonlinear Phenom. 2014, 268, 14-24. [CrossRef]

18. Avrutin, V.; Morcillo, J.D.; Zhusubaliyev, Z.T.; Angulo, F. Bubbling in a power electronic inverter: Onset, development and detection. Chaos Solitons Fractals. 2017, 104, 135-152. [CrossRef]

19. Lei, B.; Xiao, G.; Wu, X.; Ka, Y.R.; Zheng, L. Bifurcation analysis in a digitally controlled H-bridge grid-connected inverter. Int. J. Bifurc. Chaos 2014, 24, 1450002. [CrossRef]

20. Benadero, L.; Ponce, E.; El Aroudi, A.; Torres, F. Limit cycle bifurcations in resonant LC power inverters under zero current switching strategy. Nonlinear Dyn. 2018, 91, 1145-1161. [CrossRef]

21. Albea, C.; Gordillo, F. Estimation of the region of attraction for a boost DC-AC converter control law. In Proceedings of the IFAC Symposium on Nonlinear Control Systems (Nolcos), Pretoria, South Africa, 21-24 August 2007; pp. 874-879.

22. Liao, Z.-X.; Luo, D.; Luo, X.-S.; Li, H.-S.; Xiang, Q.-Q.; Huang, G.-X.; Li, T.-H.; Jiang, P.-Q. Nonlinear model and dynamic behavior of photovoltaic grid-connected inverter. Appl. Sci. 2020, 10, 2120. [CrossRef]

23. Lu, W.G.; Jing, F.; Zhou, L.; Iu, H.H.C.; Fernando, T. Control of sub-harmonic oscillation in peak current mode buck converter with dynamic resonant perturbation. Int. J. Circuit Theory Appl. 2015, 43, 1399-1411. [CrossRef]

24. El Aroudi, A.; Mandal, K.; Giaouris, D.; Banerjee, S.; Abusorrah, A.; Al Hindawi, M.; Al-Turki, Y. Fast-scale stability limits of a two-stage boost power converter. Int. J. Circuit Theory Appl. 2016, 44, 1127-1141. [CrossRef]

25. El Aroudi, A. A new approach for accurate prediction of subharmonic oscillation in switching regulators-part I: Mathematical derivations. IEEE Trans. Power Electron. 2017, 32, 5651-5665. [CrossRef]

26. El Aroudi, A. A new approach for accurate prediction of subharmonic oscillation in switching regulators-part II: Case studies. IEEE Trans. Power Electron. 2017, 32, 5835-5849. [CrossRef]

27. El Aroudi, A.; Al-Numay, M.; Calvente, J.; Giral, R.; Rodriguez, E.; Alarcón, E. Prediction of subharmonic oscillation in switching regulators: from a slope to a ripple standpoint. Int. J. Electron. 2016, 103, $2090-2109$. [CrossRef]

28. Huang, M.; Ji, H.; Sun, J.; Wei, L.; Zha, X. Bifurcation-based stability analysis of photovoltaic-battery hybrid power system. IEEE J. Emerg. Sel. Top. Power Electron. 2017, 5, 1055-1067. [CrossRef]

29. Huang, M.; Peng, Y.; Tse, C.K.; Liu, Y.; Sun, J.; Zha, X. Bifurcation and large-signal stability analysis of three-phase voltage source converter under grid voltage dips. IEEE Trans. Power Electron. 2017, 32, 8868-8879. [CrossRef]

30. Peng, D.; Huang, M.; Li, J.; Sun, J.; Zha, X.; Wang, C. Large-signal stability criterion for parallel-connected DC-DC converters with current source equivalence. IEEE Trans. Circuits Syst. II Express Briefs 2019, 66, $2037-2041$. [CrossRef]

31. Cheng, L.; Ki, W.-H.; Yang, F.; Mok, P.K.T.; Jing, X. Predicting subharmonic oscillation of voltage-mode switching converters using a circuit-oriented geometrical approach. IEEE Trans. Circuits Syst. Reg. Pap. 2017, 64, 717-730. [CrossRef]

32. Leng, M.; Zhou, G.; Zhou, S.; Zhang, K.; Xu, S. Stability analysis for peak current-mode controlled buck LED driver Based on discrete-time modeling. IEEE J. Emerg. Sel. Top. Power Electron. 2018, 6, 1567-1580. [CrossRef]

33. Zhou, S.; Zhou, G.; Zeng, S.; Xu, S.; Ma, H. Unified discrete-mapping model and dynamical behavior analysis of current-mode controlled single-inductor dual-output DC-DC Converter. IEEE J. Emerg. Sel. Top. Power Electron. 2019, 7, 366-380. [CrossRef]

34. Banerjee, S.; Verghese, G.C. Nonlinear Phenomena in Power Electronics Attractors, Bifurcations, Chaos, and Nonlinear Control; IEEE Press: New York, NY, USA, 2001.

35. Tse, C.K. Complex Behavior of Switching Power Converters; CRC Press: New York, NY, USA, 2003. 
36. Mazumder, S.K.; Nayfeh, A.H.; Boroyevich, D. Theoretical and experimental investigation of the fast- and slow-scale instabilities of a DC-DC converter. IEEE Trans. Power Electron. 2001, 16, 201-216. [CrossRef]

37. Fossas, E.; Olivar, G. Study of chaos in the buck converter. IEEE Trans. Circuits Syst. Fundam. Theory Appl. 1996, 43, 13-25. [CrossRef]

38. Giaouris, D.; Maity, S.; Banerjee, S.; Pickert, V.; Zahawi, B. Application of Filippov method for the analysis of subharmonic instability in DC-DC converters. Int. J. Circuit Theory Appl. 2009, 37, 899-919. [CrossRef]

39. Cortes, J.; Svikovic, V.; Alou, P.; Oliver, J.A.; Cobos, J.A.; Wisniewski, R. Accurate analysis of subharmonic oscillations of $V^{2}$ and $V^{2} I_{C}$ controls applied to buck converter. IEEE Trans. Power Electron. 2015, 30, $1005-1018$. [CrossRef]

40. El Aroudi, A.; Al-Numay, M.; Garcia, G.; Al Hossani, K.; Al Sayari, N.; Cid-Pastor, A. Analysis of nonlinear dynamics of a quadratic boost converter used for maximum power point tracking in a grid-interlinked PV system. Energies 2019, 12, 61. [CrossRef]

41. Jang, M.; Agelidis, V.G. A minimum power-processing-stage fuel-cell energy system based on a boost-inverter with a bidirectional backup battery storage. IEEE Trans. Power Electron. 2011, 26, 1568-1577. [CrossRef]

42. Zhu, G.; Tan, S.; Chen, Y.; Tse, C.K. Mitigation of low-frequency current ripple in fuel-cell inverter systems through waveform control. IEEE Trans. Power Electron. 2013, 28, 779-792. [CrossRef]

43. Jha, K.; Mishra, S.; Joshi, A. High-quality sine wave generation using a differential boost inverter at higher operating frequency. IEEE Trans. Ind. Appl. 2015, 51, 373-384. [CrossRef]

44. Flores-Bahamonde, F.; Valderrama-Blavi, H.; Bosque-Moncusi, J.M.; Garcia, G.; Martinez-Salamero, L. Using the sliding-mode control approach for analysis and design of the boost inverter. IET Power Electron. 2016, 9, 1625-1634. [CrossRef]

45. Lopez-Caiza, D.; Flores-Bahamonde, F.; Kouro, S.; Santana, V.; Müller, V.; Chub, A. Sliding mode based control of dual boost inverter forg grid connection. Energies 2019, 12, 4241. [CrossRef]

46. Meneses, D.; Blaabjerg, F.; García, O.; Cobos, J.A. Review and comparison of step-up transformerless topologies for photovoltaic AC-module application. IEEE Trans. Power Electron. 2013, 28, 2649-2663. [CrossRef]

47. Caceres, R.O.; Barbi, I. A boost DC-AC converter: Analysis, design, and experimentation. IEEE Trans. Power Electron. 1999, 14, 134-141. [CrossRef]

48. Sanchis, P.; Ursaea, A.; Gubia, E. Boost DC-AC inverter: a new control strategy. IEEE Trans. Power Electron. 2005, 20, 343-353. [CrossRef]

49. El Aroudi, A; Haroun, R.; Al-Numay, M.; Huang, M. Multiple-loop control design for a single-stage PV-fed grid-tied differential boost inverter. Appl. Sci. 2020, 10, 4808. [CrossRef]

50. Vazquez, N.; Almazan, J.; Alvarez, J. Analysis and experimental study of the buck, boost and buck-boost inverters. In Proceedings of the 30th Annual IEEE Power Electronics Specialists Conference. Record. (Cat. No. 99CH36321), Charleston, SC, USA, 1 July 1999; pp. 801-806.

51. Iu, H.H.C.; Zhou, Y.; Tse, C.K. Fast-scale instability in a boost PFC converter under average current control. Int. J. Circuit Theory Appl. 2003, 31, 611-624. [CrossRef]

52. Floquet, G. Sur les équations différentielles linéaires à coefficients périodiques. Ann. Sci. L'éCole Norm. SupéRieure 1883, 12, 47-88. [CrossRef]

53. Leine, R.L.; Nijemeijer, H. Dynamics and bifurcations of non-smooth mechanical systems. In Lecture Notes in Applied and Computational Mechanics; Springer: Berlin/Heidelberg, Germany, 2004; Volume 18.

(C) 2020 by the authors. Licensee MDPI, Basel, Switzerland. This article is an open access article distributed under the terms and conditions of the Creative Commons Attribution (CC BY) license (http:/ / creativecommons.org/licenses/by/4.0/). 